Archives de sciences sociales des religions

141 | janvier-mars 2008

Mémoires

\title{
Le rite comme contexte de la mémoire des origines
}

Robert R. Crépeau

\section{OpenEdition}

Journals

Édition électronique

URL : https://journals.openedition.org/assr/12552

DOI : $10.4000 /$ assr. 12552

ISSN : $1777-5825$

Éditeur

Éditions de l'EHESS

Édition imprimée

Date de publication : 1 mars 2008

ISBN : 978-2-7132-2189-7

ISSN : 0335-5985

\section{Référence électronique}

Robert R. Crépeau, «Le rite comme contexte de la mémoire des origines 》, Archives de sciences

sociales des religions [En ligne], 141 | janvier-mars 2008, mis en ligne le 29 novembre 2013, consulté le 05 juillet 2021. URL : http://journals.openedition.org/assr/12552 ; DOI : https://doi.org/10.4000/assr. 12552 


\section{Robert R. Crépeau}

\section{Le rite comme contexte de la mémoire des origines}

Il y a quelques années, alors que je travaillais auprès des Kaingang de la réserve amérindienne Xapecó au Brésil, un professeur brésilien, vivant avec sa famille sur cette réserve depuis plusieurs années, m'interrogea au sujet de l'origine des Amérindiens. À nos côtés se trouvait Vicente Fernandes Fokâe ${ }^{1}$, alors un aîné influent, grand responsable de l'organisation du Kikikoia, un important rite de secondes funérailles. Connaissant assez bien la question du peuplement des Amériques que j'aborde régulièrement dans mon enseignement universitaire, je me fis un plaisir d'informer ce professeur dont je connaissais le dévouement exemplaire pour les enfants de l'école primaire locale. Je lui fis part de l'hypothèse de la traversée du détroit de Béring par des groupes de chasseurs venus d'Asie au moment de la dernière grande glaciation, de la date approximative de cette traversée - que les archéologues situent majoritairement autour de 12000 à 15000 ans avant nos jours - et de l'arrivée en Amérique du Sud des premiers Amérindiens, environ un millénaire après leur incursion en Amérique du Nord. Imperturbable, Fokâe écouta sans dire un mot. Lorsque j'eus terminé mon exposé, il prit la parole en disant que tout cela était fort intéressant, mais qu'on devait plutôt situer cette origine après la grande inondation qui anéantit, il y a bien longtemps, la première humanité. Il raconta alors pour le bénéfice du professeur le récit de la Grande inondation :

Le déluge fut annoncé par Dieu : «Le jour où cette femme lavera un four à la rivière, il y aura une très grande inondation ". Joseph, le père de Jésus-Christ, construisit alors un bateau où il réunit un couple de chaque animal et un couple humain : une femme de la moitié kairu et son époux, un homme de la moitié kamé. L'eau monta durant un jour complet. Elle commença ensuite à baisser et se retira en trois jours. Il n'y avait plus de gens, ni d'animaux sur la terre.

Pic-bois, qui est kamé, vola le feu et le distribua. Les animaux organisèrent ensuite une fête, appelée Kikikoia, pour ceux qui périrent. Le premier à se présenter à son feu fut Caïman, Yamuyé yãgrè, qui est kamé, parce que les kamé arrivent toujours les premiers au feu. Ensuite le singe Kãyer, qui est kairu, alla à son feu. Vinrent

1. Vicente Fernandes Fokâe est décédé le 25 février 2006. Il sera désigné dans la suite du texte par son "nom de la forêt ", comme il le disait lui-même en portugais, c'est-à-dire son nom en langue kaingang : Fokâe. 
ensuite Fenenk, Tatou qui est kairu et ensuite Fòyin, Hérisson, qui est aussi kairu.

Les Kaingang apprirent les chants et les danses du Kikikoia de ces animaux.

Fokâe spécifia ensuite que ce récit démontrait que les Amérindiens arrivèrent au pays bien avant les Brésiliens et qu'ils en étaient par conséquent les premiers habitants. Il ajouta qu'il avait eu récemment confirmation de la vérité de cette histoire à la télévision.

Sur le coup, je me sentis très mal à l'aise, mais je réalisai rapidement que Fokâe ne se formalisait nullement de la situation. Il semblait au contraire concevoir ma version et la sienne comme deux possibles en affirmant toutefois, dans le contexte de son échange avec le professeur brésilien, la prééminence de sa version de l'histoire. Depuis, j'ai souvent repensé à cet événement. Au-delà de deux expressions différentes du possible et de l'autochtonie, j'en suis venu à penser que cet échange illustre des conceptions distinctes du temps et de la mémoire. Mais avant d'aller plus loin, il est indispensable de donner au lecteur quelques indications ethnologiques concernant les Kaingang.

\section{Les Kaingang du Brésil méridional ${ }^{2}$}

Les Kaingang sont membres de la vaste famille culturelle et linguistique Gé du Brésil. Atteignant aujourd'hui près de vingt mille personnes et présents dans quatre provinces du Brésil méridional (São Paulo, Paraná, Santa Catarina et Rio Grande do Sul), les Kaingang vivent en majorité dans des réserves administrées par le gouvernement fédéral brésilien. Bien que leur territoire soit facilement accessible de nos jours, les Kaingang étaient encore méconnus jusqu'à récemment d'un point de vue ethnologique. Il faut mentionner qu'au début des années 1960, ils avaient été exclus du fameux Harvard Central Brazil Project : "Because we thought (...) that their way of life was extinct. » (Maybury-Lewis, $1979: 6$ ). Or, les travaux récents montrent que les Kaingang contemporains se définissent activement par rapport à la société nationale brésilienne et qu'ils tentent de maintenir un fragile équilibre entre leur identité amérindienne et une réelle volonté de participation aux dynamiques régionales et nationales.

L'organisation sociale traditionnelle kaingang se caractérise par l'existence de moitiés, nommées kamé et kairu, entretenant entre elles une relation complémentaire et asymétrique. Chaque moitié comporte une section : votôro qui est

2. Le travail de terrain au Brésil a été financé par le Fonds québécois de recherche sur la société et la culture et le Conseil de Recherche en Sciences Humaines du Canada. Les enquêtes furent réalisées en 1993, 1994, 1995, 1998, 2003, 2004 et 2005. Je remercie particulièrement le professeur Silvio Coelho dos Santos de l'Université fédérale de Santa Catarina à Florianópolis pour son appui et ses conseils judicieux. Mes remerciements vont également aux collègues du Programa de Pos-Graduação em Antropologia Social et du Museu de Antropologia de l'Université fédérale de Santa Catarina à Florianópolis. Je tiens à exprimer toute ma gratitude aux Kaingang de Xapecó qui ont guidé avec générosité et patience mon apprentissage. 
associée à la moitié kairu et veineky, associée à la moitié kamé. L'appartenance des femmes et des hommes à la moitié ou à la section est patrilinéaire et est inscrite dans le nom attribué à l'enfant lors de sa naissance (Crépeau, 1997). Le dualisme kaingang est donc quadripartite et la possibilité de mariage d'une des sections est triple bien que le mariage préférentiel soit conçu en terme diamétral : la moitié kamé-veineky devant s'unir à la moitié kairu-votôro. Le dualisme kaingang s'exprime également dans plusieurs autres aspects de la vie rituelle et sociale, notamment au sein des nomenclatures animales, où plusieurs animaux sont conçus comme appartenant à l'une ou l'autre moitié (Haveroth, 1997 ; Nimuendajú, 1993), ainsi qu'astronomiques qui associent la moitié kamé au soleil et la moitié kairu à la lune (Crépeau, 1994).

Le mode de vie des Kaingang de la réserve Xapecó ${ }^{3}$ a beaucoup changé au cours de la seconde moitié du $\mathrm{XX}^{\mathrm{e}}$ siècle suite à la déforestation quasi-totale de leur territoire (Santos, 1981). Les Kaingang pratiquent l'agriculture : le maïs, la fève, le soja et le riz sont les principaux cultigènes produits pour la consommation locale et le marché. Le travail salarié constitue un complément important de revenu pour plusieurs individus œuvrant dans les grandes propriétés terriennes et les usines de la région. De plus, les personnes âgées de la réserve bénéficient de la pension de vieillesse accordée aux petits producteurs agricoles par le gouvernement fédéral. Toutes ces sources de revenu sont modestes mais font en sorte que les Kaingang de Xapecó participent à l'économie régionale en tant que petits producteurs agricoles, ouvriers salariés et petits consommateurs. Il n'est pas rare de les croiser dans les supermarchés de Xanxêre, une ville d'environ quinze mille habitants située à une vingtaine de kilomètres de la réserve.

Ce tableau économique était fort différent, il y a cinquante ans à peine. À cette époque, les Kaingang pratiquaient une économie mixte basée sur l'horticulture sur brûlis, la chasse, la pêche et la cueillette dans un milieu forestier entrecoupé de savanes. Cette région de hauts plateaux au climat tempéré était dominée par l'araucaria angustifolia (le pinheiro en portugais), une espèce de pin produisant en abondance un fruit consommé par les Kaingang qui se réunissaient dans les pinèdes où convergeaient également plusieurs espèces d'oiseaux et de mammifères comestibles.

\section{Le Kikikoia ou la « Fête des morts "}

Jusqu'à récemment, la vie rituelle des Kaingang de la réserve Xapecó comportait un temps fort constitué par la réalisation annuelle du Kikikoia, également désigné dans la littérature anthropologique par l'expression «Fête des morts » (Baldus, 1937). Il s'agit d'un rite de secondes funérailles, qui ne comportaient cependant

3. Il sera question désormais de la réserve Xapecó (Terra Indígena Xapecó), située dans la province de Santa Catarina, où a été effectuée en bonne partie notre enquête de terrain. Cette réserve de quinze mille hectares est habitée par environ quatre mille personnes. 
pas la pratique du double enterrement. Le terme «Kiki» désigne l'hydromel fabriqué pour la réalisation de ce rituel et consommé en grande quantité, alors que koia signifie littéralement " manger ensemble ». Abandonnée en 1946, suite à de fortes pressions de la part des autorités brésiliennes, le rituel du Kikikoia a repris en 1976 (Veiga, 2000) à Xapecó, qui fut l'unique lieu de sa réalisation jusqu'en 2001. Il est depuis retombé dans l'oubli.

Le Kikikoia constituait le lieu paradigmatique de la mise en scène de la complémentarité existant entre les groupes de parenté, complémentarité qu'il réitérait et actualisait en donnant à voir l'asymétrie constitutive des relations réciproques entre les moitiés kamé et kairu. Ce rituel élaboré était considéré comme le nœud de leur identité par les Kaingang d'obédience catholique qui le réalisaient jusqu'à tout récemment. En effet, il permettait d'affirmer leur singularité et de donner à voir leur amérindianité en relation : 1 - à une société régionale qui ne daigne pas toujours reconnaître leur statut identitaire distinct et 2 - à l’omniprésence des Églises évangéliques au sein de leurs réserves et à la montée du pentecôtisme qui menacent la continuité de la tradition.

Le Kikikoia se déroulait au début de l'hiver austral, caractérisé dans cette région par quelques gelées nocturnes et de rares chutes de neige d'à peine quelques centimètres. C'est la période de l'année qui marque la fin des récoltes et précède les plantations qui débutent en juin et juillet. Il s'agit par conséquent d'une période d'abondance durant laquelle le maïs, le haricot et le riz ne manquent jamais. C'est également à cette période de l'année que le pin araucaria produit son fruit, le pignon, consommé en abondance à partir de mars (Veiga, 1994 : 28).

Les préparatifs du Kikikoia débutaient plusieurs semaines avant sa réalisation et découlaient de la demande formelle de sa réalisation par les proches de personnes décédées. La première étape du rituel consistait à abattre un pin de fort diamètre, une opération effectuée en présence d'au moins deux officiants, un de chaque moitié, qui performaient des chants destinés à l'arbre (Veiga, 1994 : 168). Les kamé initiaient toujours ces chants suivis des kairu, un ordre scrupuleusement respecté tout au long du rituel. Les Kaingang expliquent cet important aspect de la mise en scène rituelle en disant que les kamé sont toujours premiers parce qu'ils possèdent plus de force que les kairu. Ils affrontent donc les premiers les dangers inhérents au contact avec les esprits des morts ${ }^{4}$. Le pin araucaria atteint une trentaine de mètres de hauteur à maturité et se caractérise par des rameaux alternés formant une croix. L'arbre est associé à la moitié kamé. Son tronc était transformé en une auge oblongue dans laquelle était préparé l'hydromel. Les Kaingang affirmaient que cette auge était l'équivalent symbolique d'un défunt lors du Kikikoia.

4. Cette prééminence des kamé est énoncée explicitement par Fokâe dans son récit de la Grande inondation cité en introduction. 
La phase finale du rituel consistait en deux moments distincts. Le premier durait du coucher au lever du soleil. La nuit se passait en chants, musique, prières, pleurs, rires et conversations. Les membres de chacune des moitiés se regroupaient autour de six feux alignés selon un axe est-ouest : les kairu à l'est et les kamé à l'ouest. S'adressant aux esprits des défunts (veinkupri), chaque moitié se trouvait à occuper la position des esprits de la moitié opposée.

Tous les participants devaient arborer les peintures faciales propres à leur moitié d'appartenance. Les Kaingang disent que la peinture faciale constitue une protection contre les esprits des défunts qui auraient le désir d'emmener les vivants avec eux. Pour la même raison, les feux sont alimentés uniquement avec des nœuds de pin araucaria qui brûlent longtemps tout en dégageant une forte chaleur. Les nœuds offriraient une protection contre les esprits puisque, ainsi qu'il est mentionné ci-dessus, les branches de l'arbre dont ils sont issus forment une croix. On entrevoit quelques-unes des influences judéo-chrétiennes du rituel qui vont également apparaitre lors de la procession vers le cimetière qu'entreprenaient les moitiés à la levée du jour. Les kamé prenaient la tête de cette procession, suivis à bonne distance par les kairu. Chaque moitié transportait les croix de bois qui étaient plantées sur la tombe des défunts de la moitié opposée.

Le cimetière kaingang était divisé en deux sections. On y enterrait les kamé du côté élevé et les kairu dans la moitié basse du cimetière. Les officiants kamé y entraient les premiers et effectuaient les chants et opérations rituelles destinés aux défunts kairu. Les officiants kairu faisaient ensuite de même pour les défunts kamé. Les officiants de chaque moitié plantaient les croix et retiraient le tipãkri, un rameau de pin pour les kamé ou de feuillu pour les kairu disposé sur la tombe de chaque défunt au moment des premières funérailles. Le tipãkri était alors lancé à l'extérieur de l'enceinte du cimetière dans la direction où devait cheminer l'esprit du défunt ainsi libéré du lieu où il était confiné jusqu'au Kikikoia.

Le retour vers les feux de la veille, depuis le cimetière, s'effectuait séparément : les kamé marchant toujours devant les kairu. À l'entrée du village, chaque moitié était accueillie par un officiant de la moitié opposée qui distribuait généreusement de l'hydromel à chacun des participants. Les moitiés se dirigeaient ensuite à l'emplacement des feux et de l'auge où elles dansaient, tout d'abord séparément, pour ensuite se rapprocher progressivement l'une de l'autre. Ce rapprochement était initié par la moitié kamé. La séparation et la distance qui avaient été maintenues entre les moitiés tout au long du rituel, étaient abolies par cette fusion chorégraphique. Les moitiés ne formaient plus qu'un seul ensemble, une vague humaine, où elles se confondaient pour la première fois. Les participants se réunissaient ensuite autour de l'auge qu'ils vidaient jusqu'à la dernière goutte de son contenu d'hydromel avant de la renverser et de la frapper avec des bâtons et des pierres pour signifier la clôture du rituel. 


\section{Quelques éléments d'analyse}

La description qui précède n'offre aux lecteurs qu'un aperçu sommaire du rituel tout en respectant ses grandes lignes ${ }^{5}$. J'aimerais à présent suggérer quelques éléments d'analyse du Kikikoia en lien avec son texte fondateur, le récit de la Grande inondation présenté en introduction. En éliminant tous les humains à l'exception d'un couple primordial, la Grande inondation abolit le cycle des échanges matrimoniaux et de la réciprocité. En effet, en vertu du principe de descendance patrilinéaire en vigueur chez les Kaingang, les descendants de ce couple primordial, un homme kamé et une femme kairu, appartiennent à la moitié kamé. À la discontinuité des moitiés, le récit substitue le continu de la consanguinité. Selon d'autres versions de ce récit, c'est de ce continu que fut engendrée la nouvelle humanité issue d'ancêtres kamé qui réinstaurèrent les divisions sociologiques antédiluviennes (Crépeau, 1997).

Procédant à rebours du mythe qui instaure une unité qu'il scinde ensuite, le Kikikoia met en scène, à travers le traitement réciproque des morts, le dualisme social, l'asymétrie et la complémentarité des moitiés qu'il maintient tout au long $\mathrm{du}$ rite et qu'il abolit uniquement en fin de parcours lors de la danse finale. Le rituel travaille ainsi à partir des divisions sociologiques - réduites formellement aux deux moitiés constitutives de la société - et il met en scène l'unité postdiluvienne qu'il représente par la fusion chorégraphique des moitiés lors de la danse finale où ces dernières ne forment plus qu'une seule vague humaine.

\section{Mémoire et rituel}

Claude Lévi-Strauss (1983) a montré, à l'aide de mythes grecs et amérindiens, que le motif de l'oubli caractérise fréquemment les mythes fondateurs de pratiques rituelles. Il souligne que les affinités entre mythes grecs et amérindiens s'étendent jusqu'aux métaphores et excluent un rapprochement historique et géographique. L'oubli serait plus qu'un artifice ou une «ficelle » à laquelle le narrateur recourt arbitrairement pour remettre en marche une intrigue. Il serait une véritable catégorie de la pensée mythique. Dans les mythes où il intervient, le motif de l'oubli sert à fonder un système rituel ou des interdictions et des prescriptions rituelles (Levi-Strauss, 1983 : 258-259). Plus précisément, les récits amérindiens cités par Lévi-Strauss sont des mythes rendant compte de l'origine de la société et de ses institutions. Ainsi, dans les contextes hidatsa qu'il a analysés (1983 : 253), le mythe jette les bases d'un calendrier cérémoniel, c'est-à-dire d'un ordre sériel.

Lévi-Strauss définit l'oubli comme un défaut de communication avec soimême : "car oublier, c'est manquer de dire à soi-même ce qu'on aurait dû pouvoir se dire » (1973 : 230). L'oubli formerait système avec le malentendu, défini

5. Pour une description détaillée du Kikikoia, voir Crépeau, 2006. 
comme défaut de communication avec autrui, et avec l'indiscrétion, un excès de communication avec autrui. La dernière permutation correspond à un excès de communication avec soi-même qui se manifeste sous la forme de la nostalgie. Lévi-Strauss n'insiste pas sur ce dernier motif, malgré le fait que la nostalgie du héros dans la geste d'Asdiwal constitue un important moteur de l'action du récit. Il indique cependant : "Après une conférence faite en février 1973 à Vancouver, où j'avais exposé cette interprétation (...), Mlle Hilda Thomas ${ }^{6}$ a suggéré que la nostalgie, qui est le contraire de l'oubli, pourrait être définie comme excès de communication avec soi-même (...) ${ }^{7}$.

Tableau des permutations formées par ces quatre motifs (Adapté d'après Lévi-Strauss, $1973: 230)$ :

\begin{tabular}{|c|c|c|c|c|}
\hline & Indiscrétion & Malentendu & Oubli & Nostalgie $^{8}$ \\
\hline $\begin{array}{c}\text { Excès / Défaut } \\
+\quad-\end{array}$ & + & - & - & + \\
\hline $\begin{array}{c}\text { Autrui / Soi } \\
+\quad-\end{array}$ & + & + & - & - \\
\hline
\end{tabular}

Lévi-Strauss en déduit que « la fonction propre du rituel est (...) de préserver la continuité du vécu (...), continuité que vient briser l'oubli dans l'ordre mental : nous le reconnaissons nous-mêmes en parlant de "trou de mémoire" " (1983: 259).

Continuité du vécu par rapport à quoi ? En fait, ces quatre modes de communication avec soi-même et avec autrui possèdent des connotations négatives et constituent des ruptures, par défaut ou excès, par rapport à une communication normale et positive. Il est possible d'associer ces connotations négatives à une défaillance ou un déficit de la mémoire dans les cas de défaut de communication (oubli et malentendu) ou à un trop plein de mémoire en ce qui concerne les excès de communication (indiscrétion et nostalgie - à laquelle on pourrait ajouter le regret, le remords, le repentir).

Plusieurs mythes recourant aux motifs du défaut de communication avec soimême ou avec autrui instituent un ordre qui découle de la destruction préalable

6. Hilda Thomas († 2005) a enseigné la littérature à l’Université de Vancouver.

7. Il est intéressant de noter que Lévi-Strauss ne reprend pas cette quatrième catégorie dans son texte de 1983. Il la mentionne cependant dans un autre texte du même volume (1983, pp. 230-1) : "Or, j’ai montré naguère que toutes les versions tsimshian du mythe d'Asdiwal mettent en œuvre des modalités diverses de la communication : indiscrétion, comme excès de communication avec autrui ; malentendu, comme défaut de communication, aussi avec autrui ; oubli, comme défaut de communication avec soi-même; et peut-être nostalgie, comme excès de communication avec soi-même (Anthropologie structurale deux : 229-213)».

8. La nostalgie n’apparaît pas au tableau publié par Lévi-Strauss en 1973. 
d'un monde antérieur conçu comme stérile et irrécupérable, destruction sacrificielle rendant possible l'émergence et la genèse du monde actuel. C'est cette émergence d'une fécondité et d'une fertilité essentielles à la continuité du vécu que met en scène le Kikikoia en actualisant le souvenir de la Grande inondation relaté par le mythe.

\section{La Grande inondation}

Selon le récit de Fokâe, la Grande inondation fut annoncée par Dieu et provoquée « le jour où une femme lava un four à la rivière ", une phrase mystérieuse dont le sens n'est pas explicité. On pourrait y voir un oubli de l'avertissement divin, bref un défaut de communication de cette protagoniste anonyme avec ellemême, mais on ne peut également écarter la possibilité d'un malentendu de la part de cette femme malgré l'avertissement divin, et donc d'un défaut de communication avec autrui. Dans les deux cas, il s'agirait d'une pathologie de la communication qui provoque un événement remontant au temps des origines. Cet événement est au fondement d'un rituel que les animaux réaliseront pour la première fois après la Grande inondation. Le récit précise que c'est des animaux que les Kaingang apprirent à réaliser le Kikikoia.

Comme l'a montré Lévi-Strauss, la preuve que les motifs de l'oubli, du malentendu, de l'indiscrétion et de la nostalgie forment système est fournie par "l'alternance ou le cumul de ces motifs dans les variantes d'un même mythe " (1983 : 253). Je tenterai pour ma part de vérifier, de façon préliminaire, en quoi ces mêmes motifs forment système non pas dans les variantes d'un même mythe, mais dans celles d'une même catégorie de mythes : les récits fondateurs, c'est-àdire d'origine, d'émergence et de destruction cosmique. Mon analyse se limitera toutefois à quelques récits sud-américains et au thème de la femme lavant un four à la rivière afin de mieux cerner et, éventuellement, confirmer sa relation avec les motifs de l'oubli et du malentendu.

Un mythe intitulé « l'incendie universel et le déluge », recueilli chez les Tembé du nord du Brésil, des Amérindiens de la famille linguistique tupi-guarani, nous permettra de débuter notre parcours :

Il y a bien longtemps, un homme s'approcha d'un enfant qui jouait seul. Il lui donna une torche allumée en lui ordonnant de l'éteindre dans l'eau de la rivière et il disparut aussitôt. L'enfant mit la torche à l'eau qui s'enflamma aussitôt. Au début l'eau brûla et par la suite la terre commença à dégager de hautes flammes. Le feu courut sous terre et fit irruption sur la place d'un village. La terre s'effondra à cet endroit. Une femme enceinte se réfugia avec un jeune garçon dans une bananeraie qui ne pouvait être détruite par le feu. L'incendie anéantit toute l'humanité. Après son extinction, les deux survivants sortirent de leur refuge. Dans l'immense brûlis, ils trouvèrent cinq boutures de manioc qu'ils conservèrent soigneusement. Il se mit alors à pleuvoir durant plusieurs jours et nuits. Les deux eurent très faim. Enfin, l'eau se retira lentement et 
lorsque la terre fut dégagée, ils plantèrent les boutures de manioc. La femme donna naissance à une fille; de cette dernière et du garçon se recréa l'humanité (Baldus, $1960: 118)^{9}$.

Ce récit tembé transforme plusieurs éléments du mythe kaingang. Au thème de la femme et son four à la rivière est substitué celui d'un enfant au sexe indéfini qui enflamme l'eau et la terre avec une torche incandescente reçue des mains d'un homme anonyme qui s'empresse de disparaître. Le motif de l'oubli est remplacé ici par celui de l'obéissance, aveugle ou naïve pourrait-on dire, à la demande d'un inconnu. En omettant de se questionner avant d'agir, l'enfant pèche par un défaut de communication avec soi-même comparable à l'oubli.

Proches des Kaingang sur le plan culturel et social, les Bororo du Brésil central possèdent plusieurs récits relatant une inondation primordiale. L'Encyclopédie Bororo d'Albisetti et Venturelli rapporte le récit suivant :

Lors d'une pêche collective, les compagnons du fils de Meríri Póro, resté au village pour fabriquer des flèches, se moquèrent de lui et de sa mère en lui offrant un poisson qu'ils associèrent au sexe de cette dernière. Informé de ce fait par son fils, Meríri Póro en fut profondément offensé et courut au lieu de pêche où il ne trouva qu'un esprit. Sous l'emprise de la colère, il le flécha. Immédiatement, la rivière se mit à déborder dans un grondement effrayant. Meríri Póro eut à peine le temps de prendre un tison et se réfugier au sommet de la montagne voisine, nommée Toroári, avant que toute la terre ne soit inondée. Voyant que les eaux continuaient à monter, il réchauffa des pierres qu'il jetta à l'eau qui, au contact de ces dernières, s'évapora retrouvant graduellement son niveau normal. Meríri Póro vit qu'il était le seul Bororo ayant survécu à l'inondation. Après avoir longtemps marché, il rencontra un cerf Pobógo femelle dont il fit son épouse. De leur union, naquirent d'abord plusieurs enfants aux allures animales, mais petit à petit les enfants eurent une allure humaine. C'est ainsi que Meríri Póro engendra de nouveau les Bororo (Albisetti et Venturelli, 1969 : 3-15) ${ }^{10}$.

Transformant l'annonce de Dieu liant une femme lavant un four à la rivière et l'avènement de la Grande inondation, ce récit bororo met en scène un homme provoquant l'événement destructeur en fléchant un esprit alors qu'il croit punir ceux qui s'étaient moqués de son épouse. Réfugié au sommet d'une montagne, l'homme arrête la crue des eaux à l'aide de pierres chauffées. Nous avons les contrastes suivants entre les récits kaingang et boboro : femme qui lave/homme qui flèche ; esprit annonciateur/esprit agressé ; four lavé au niveau de la rivière qui provoque l'inondation/pierres chauffées au sommet de la montagne qui arrêtent l'inondation. Le motif de l'oubli est substitué ici par celui de la colère qui amène Meríri Póro à flécher un esprit en lieu et place de ceux qui ont insulté son épouse. On peut définir la colère comme un défaut de communication avec soi-même. En effet, quelqu'un qui s'emporte ou agi sous l'emprise de la colère est coupable d'un défaut de communication avec soi-même en ce qu'il omet de réfléchir avant de poser son geste. La colère serait donc ici un équivalent de l'oubli.

\footnotetext{
9. J'ai traduit et résumé le texte portugais.
}

10. Traduction et résumé du texte portugais. 
Chez les Jivaro d'Amazonie péruvienne et équatorienne, le thème de la femme qui lave un four à la rivière se transforme en celui d'une épouse qui frappe et brûle un esprit aquatique féminin que son mari cache dans un panier. Dans une version shuar citée par Pellizzaro (1980), un homme cache son épouse spirituelle enceinte, un esprit aquatique Tsunki à la belle et longue chevelure, dans un panier qu'il range sur une poutre élevée de la maison en interdisant à ses deux épouses terrestres de l'ouvrir. Celles-ci désobéissent et y trouvent l'esprit aquatique sous la forme d'un petit serpent qu'elles frappent et brûlent avec des tisons ardents. L'épouse spirituelle tombe à l'eau et retourne auprès de son père qui, en représailles, provoque le déluge et lâche ses anacondas contre les humains. L'époux et sa fille terrestre survivent en se réfugiant à la cime d'un palmier au sommet d'une montagne. Le récit conclut que c'est d'eux que descendent les Shuar.

Le four du récit kaingang se transforme en tisons, comme dans le mythe bororo, avec lesquels les femmes brûlent l'esprit aquatique suite à un double défaut de communication : 1 - elles désobéissent à l'interdiction d'ouvrir le panier ; 2 - elles ignorent que le serpent est en réalité un esprit ; ce qu'elles auraient dû, en fait, déduire du comportement très particulier de leur mari sur lequel s'attardent longuement les diverses versions de ce récit jivaro. Si la désobéissance est un acte comparable au malentendu et constitue un défaut de communication avec autrui, l'ignorance constitue, dans le contexte de ce récit, un défaut de communication avec soi-même et donc un équivalent de l'oubli. Soulignons que ce récit jivaro est lié à l'origine des rites et des pouvoirs chamaniques dont les esprits aquatiques Tsunki sont la source primordiale selon les Jivaro (Crépeau, 1988) ${ }^{11}$.

Les Yagua d'Amazonie péruvienne, un groupe de la famille linguistique pebayagua, rapportent le récit d'un incendie cosmique :

Une vieille femme disait que le monde allait finir dans moins d'une lune. Elle ne cessait de mettre en garde les gens de la terre (...) Mais les gens ne prirent pas au sérieux les présages de la vieille femme (...) ils ne la croyaient pas (...) personne ne croyait vraiment à la fin du monde. La vieille femme commença à creuser au pied d'une colline (...) jusqu'à ce qu'elle jugeât l'abri sûr. Elle l'emménagea comme sa propre maison et y stocka des aliments, des semences et des jeunes pousses. Quand l'heure approcha, l'abri était fin prêt. La vieille femme dit alors à son mari : «Entrons! C'est la fin." L'ouverture fut soigneusement bouchée. Ils passèrent ainsi l'année du déluge (...) " huñídanu [le créateur] nous a épargnés pour que l'on raconte aux gens... et pour leur donner les plantes cultivées. "(Chaumeil, 1983: 153).

Ce mythe transforme plusieurs aspects des récits précédents, à commencer par l'inondation qui devient un incendie cosmique - qualifié de " déluge » par le narrateur... - qu'une vieille femme annonce sans que personne ne la croie. Avec son mari, elle se réfugie dans un abri creusé au pied d'une colline - et non au sommet d'un palmier et d'une montagne comme dans le récit shuar (Crépeau,

11. Les rites chamaniques dont le rôle est justement de faire sens des conflits interindividuels chez les Jivaro. 
1994) ${ }^{12}$. Le récit ne précise pas la cause de l'incendie mais indique que le créateur a épargné le couple pour lui permettre de donner les plantes cultivées qu'il a sauvées de la destruction en les stockant sous terre. Dans le contexte de ce récit yagua, le fait de ne pas croire l'annonce de la vieille femme constitue un défaut de communication avec autrui, un acte comparable au malentendu.

Pour conclure ce parcours, j’examinerai deux récits des Kayapó du Brésil, une société de la famille linguistique Gé à laquelle appartiennent les Kaingang :

Un homme oublia un objet dans un camp abandonné. Il retourna sur ses pas pour le récupérer. En route, il aperçut des esprits masculins assis sur leur longue chevelure qui flottaient sur une rivière. Il en flécha un qui tomba à l'eau en poussant un grand cri. La rivière se mit aussitôt à déborder et à inonder les berges. Effrayé, l'Indien s'enfuit vers son village, mais l'eau le rattrapa. Il eut tout juste le temps de mettre un enfant à l'abri dans un contenant et de monter dans un arbre situé au sommet d'une montagne. La plupart des humains qui se réfugièrent dans les arbres se transformèrent en diverses espèces animales et en abeilles. L'enfant caché dans le récipient se transforma en crapaud. Seul l'homme qui avait fléché l'esprit demeura humain. Il est l'ancêtre des Kayapó (Wilbert, 1978 : 122-123) ${ }^{13}$.

En lieu et place de la femme qui lave un four à la rivière, le motif de l'oubli ouvre explicitement ce récit kayapó. Un homme ayant oublié un objet dans un camp aperçoit un esprit aquatique masculin - à la longue chevelure rappelant celle de l'épouse spirituelle du récit shuar - qu'il flèche provoquant ainsi la grande inondation. Comme dans le récit bororo, cet homme est l'unique survivant et à l'origine de l'humanité kayapó actuelle.

L'action du dernier récit, provenant également des Kayapó, se déroule après la Grande inondation. Il diffère donc des précédents à plusieurs niveaux. Son intérêt est de réintroduire le thème de l'annonce divine du déluge présent dans le récit kaingang narré par Fokâe :

Peu de temps après la grande inondation, plusieurs personnes marchaient en récoltant les poissons jonchant encore le sol. Elles arrivèrent à une plantation de manioc. Les tubercules étaient pourris pour la plupart à cause de l'inondation, mais quelques-uns encore comestibles furent grillés et mangés avec le poisson. Après le repas, les personnes se rendirent à l'étang pour boire. L'arc-en-ciel leur apparut. Lorsqu'elles l'approchèrent, l'arc-en-ciel se transforma en une femme au ventre rempli d'eau. Cette dernière leur donna de l'eau en disant : "Lorsque vous voudrez de l'eau, adressez vous à moi. Aussi longtemps que vous me verrez au début de la saison des pluies, vous vivrez en paix, car rien ne vous arrivera. Mais le jour où je ne montrerai plus, la terre sera inondée et vous allez tous mourir»(Wilbert, $1984: 43)^{14}$.

Ce récit transforme la figure divine masculine kaingang qui annonce la Grande inondation par arc-en-ciel, un personnage céleste féminin. Ce dernier n'est

12. Dans d'autres versions du mythe kaingang de la Grande inondation, les survivants se réfugient également au sommet d'un palmier et d'une montagne et non pas dans un bateau analogue à l'arche biblique.

13. J'ai traduit et résumé le texte anglais.

14. Ma traduction. 
plus associé au feu - par un four, une torche, un tison ou l'incendie cosmique mais plutôt à l'eau qu'il contrôle pour le bénéfice des humains. Le récit kayapó annonce le retour de la Grande inondation par la disparition future d'arc-en-ciel. Aucun défaut ou excès de communication ne semble en cause. Cependant, comme l'a souligné Lévi-Strauss : "En Amérique du Sud, l'arc-en-ciel possède une double signification. D'une part, et comme ailleurs, il annonce la fin de la pluie ; d'autre part, on le tient pour responsable des maladies et de divers cataclysmes naturels. Sous le premier aspect, l'arc-en-ciel opère la disjonction du ciel et de la terre, précédemment unis par l'intermédiaire de la pluie. Sous le second aspect, il remplace une conjonction normale et bienfaisante par une conjonction anormale et malfaisante : celle qu'il assure lui-même entre le ciel et la terre, en se substituant à l'eau. » 1964 : 252). Transposée en termes de communication, une conjonction anormale et malfaisante est comparable à un défaut ou à un excès de communication, mais seul un examen de l'ensemble des versions de ce récit permettrait de le confirmer avec certitude.

Les mythes que nous avons brièvement analysés ont révélé d'autres motifs en rapport de transformation avec l'oubli et le malentendu : l'obéissance aveugle (récit tembé), la colère aveugle (récit bororo), la désobéissance et l'ignorance fautive (récit jivaro), ainsi que l'incrédulité (mythe yagua). Ces cinq motifs forment système dans une même catégorie de récits de destruction cosmique par l'eau ou/et par le feu et correspondent tous à un défaut de communication avec soi-même (l'obéissance aveugle, la colère aveugle, l'ignorance fautive) ou avec autrui (désobéissance, incrédulité). Il me semble significatif qu'aucun des récits analysés ne pose l'indiscrétion ou la nostalgie comme déclencheur de la destruction cosmique ${ }^{15}$.

Le propre du déluge ou de l'incendie cosmique est d'engendrer l'union des éléments : eau, terre et feu ainsi que la conjonction des domaines : terrestre, céleste et aquatique. C'est ce qu'illustre le récit tembé où l'eau et la terre s'enflamment au contact de la torche. L'incendie se propage également sous terre et provoque son effondrement, unissant ainsi les domaines terrestre et aquatique au domaine chtonien.

15. Le motif de l'oubli comme défaut de communication avec soi-même apparait inversé dans la Genèse sous la forme d'un excès de communication avec soi-même. En effet, le texte de la Bible affirme que Yahvé provoque le déluge parce qu'il « regrette d'avoir fait l'adam sur la terre et se tourne douleur vers son cœur » (La Bible. Nouvelle traduction, 2001, Gn 6,6) ou "se repentit d'avoir fait l'homme sur la terre et il s'affligea dans son cœur" (La Bible de Jérusalem, 1981, Gn 6,6). Alors que : "Noé suit toutes les instructions de Yhwh " ou "Noé fit tout ce que Yahvé lui avait commandé. » (Gn 7,5), bref n’oublie rien. De plus à la sortie de l'arche, Noé sacrifie les animaux purs sur l'autel à Yahwé : «... il prit de tous les animaux purs et de tous les oiseaux purs et offrit les holocaustes sur l'autel. " $(\mathrm{Gn} 8,20)$. Alors que dans le récit de Fokâe, ce sont les animaux qui réalisent le premier Kikikoia destiné aux victimes de la grande inondation. 
Or, le Kikikoia est construit de façon à unir les moitiés et les sections ainsi que le masculin et le féminin. Tout cela est conçu comme essentiel à la réussite du rite. L'importance du rôle des femmes dans son déroulement est remarquable. En effet, les officiants nommés penk - qui sont en majorité des femmes - jouent un rôle essentiel tout au long du rituel en assistant les officiants-chanteurs et en procédant à des opérations cruciales pour sa réussite, notamment l'enlèvement et l'expulsion du típãkri du cimetière, gestes qui, comme je l'ai indiqué ci-dessus, permettent la libération de l'esprit du défunt qu'on conçoit dès lors comme cheminant vers son lieu de repos.

\section{Le rite comme contexte de la mémoire du mythe}

Henri Atlan a fait remarquer qu'il existe une différence entre « se souvenir » et «ne pas oublier " ${ }^{16}$. Le souvenir n'est pas l'exact opposé de l'oubli : "On peut ne pas avoir oublié et pourtant ne pas se souvenir : c'est resté enfoui quelque part. " Je l'ai sur le bout de la langue, comme on dit parfois. «L'inverse est vrai : on peut avoir oublié et pourtant se souvenir. (...) On se souvient de quelque chose qui a en fait été oublié : c'est ce qu'actualise le rite » (2003 : 307). Il s'agit du souvenir du temps des origines, un temps hors du temps auquel chaque rite, pris individuellement, se réfère et remonte. Le rite constitue en quelque sorte un " rendez-vous ", ce que rend bien le terme kaingang Kikikoia, qui signifie manger ensemble : «koia » le «kiki », c'est-à-dire l'hydromel.

Selon Atlan, notre mémoire comporte schématiquement deux fonctions distinctes : "l'une de stockage, mise en mémoire et conservation à la façon d'une écriture, et l'autre de rappel $»(2003: 309)$. Cette dernière fonction implique que le contenu effectif du passé est affecté par le contexte présent du rappel. Ce dernier transforme ce contenu en le projetant dans le présent et le futur, bref l'actualise.

Le mythe a justement comme caractéristique de relater un événement qui n'est plus soumis à une exigence de conservation exacte et précise, c'est-à-dire en tant que souvenir individuel. Il relève de la mémoire collective et lorsqu'il fonde un système rituel, il est lié aux rendez-vous que la collectivité désire honorer et dont elle souhaite collectivement se souvenir, bref ne pas oublier. Le récit de l'origine se déroule dans un temps hors du temps; l'événement décrit se caractérise justement par une intemporalité fondatrice du temps présent et du futur.

Considérations en apparence abstraites, mais qui étaient pourtant fort concrètes pour mes interlocuteurs kaingang, particulièrement Vicente Fernandes Fokâe à l'époque de notre conversation concernant l'origine des Amérindiens du

16. En relation au texte de Lévi-Strauss évoqué ci-dessus, "se souvenir " correspond de façon symétrique et inverse aux formes d'excès de la mémoire que sont l'indiscrétion et la nostalgie alors que "ne pas oublier " correspond de façon symétrique et inverse aux formes défectueuses de la mémoire que constituent le malentendu et bien sûr l'oubli. 
Brésil. En effet, la reprise du Kikikoia en 1976 coïncida avec un regain de vigueur des luttes pour la préservation de la réserve de Xapecó dont le territoire initial, délimité au début du $\mathrm{XX}^{\mathrm{e}}$ siècle, a été amputé de plusieurs milliers d'hectares suite à l'intrusion de colons brésiliens. Mes hôtes kaingang ont été tout à fait explicites à ce sujet en liant sans cesse la réalisation du Kikikoia à un devoir de mémoire en relation directe avec la question territoriale, plus précisément à la préservation des territoires qu'ils possèdent actuellement ainsi qu'à la lutte pour la récupération d'une vaste portion, dite Toldo Imbu, de leur réserve initiale qui fut vendue frauduleusement à des colons en 1940 (D’Angelis et Fokâe, 1994).

\section{Conclusion}

Robert Hertz a jadis souligné que les secondes funérailles constituent toujours un événement au "caractère collectif prononcé » et qu'elles supposent: "une concentration du corps social sur lui-même; mais, en ce cas ce n'est pas la famille ni même le village, c'est la nation qui intervient directement pour réintégrer les morts dans la communion sociale. Cet acte prend dès lors une signification politique : en mettant en commun tous leurs morts, les divers groupes domestiques et locaux qui forment l'unité supérieure prennent conscience des liens qui les unissent et par la suite ils les entretiennent ; en constituant la société des morts, la société des vivants se recrée régulièrement elle-même " (1970:63). Ainsi, le rite ne peut pas être seulement répétition du passé, mais engendrement d'un présent et d'un futur. L'enjeu profond de tout rituel est l'actualisation au présent d'une certaine mémoire : le passé et d'une mémoire encore incertaine : le futur.

Les Kaingang affirment que la réalisation du Kikikoia ne doit comporter aucune erreur sous peine d'entraîner de graves conséquences dans l'année qui suit. On mentionne surtout que des décès découleront des erreurs commises. On ne peut s'empêcher de voir un parallèle entre cette conception d'un rituel réalisé sans erreur et les motifs de l'oubli et du malentendu que les mythes posent comme précurseurs d'événements destructeurs et mortifères. Se souvenir des défunts sans défaillance de la mémoire au cours de la réalisation effective du rituel place donc le Kikikoia dans un rapport symétrique et inverse avec l'événement primordial qui le fonde. En effet, selon les Kaingang, les erreurs rituelles découlent précisément de défauts de communication avec soi-même ou avec autrui : problème dans la mémorisation des chants ${ }^{17}$ ou de la séquence des opérations rituelles. Par exemple, une erreur fréquemment commise concerne la direction dans laquelle est lancé le típãkri d'un défunt. En effet, le típãkri d'un défunt kamé doit être lancé en

17. Un jeune chanteur rituel me confia, en 2005 , ne pas se sentir prêt à prendre la relève en raison de ses difficultés à mémoriser une séquence particulièrement difficile d'un chant que lui transmit son défunt père - et cela malgré le fait qu'il possède l'enregistrement sur cassette d'une performance de ce dernier. 
direction est et celui d'un kairu en direction ouest ; c'est dans cette direction que l'esprit veinkupri cheminera vers son lieu de repos, disent les Kaingang.

Le temps des origines est décrit dans nos récits amérindiens comme un temps de fécondation, de fécondité et de naissance qui succède à la dévastation et à la mort. L'actualisation rituelle de la mémoire du passé et des temps futurs est porteuse de projet et créatrice de significations, justement parce qu'elle établit un lien entre la mémoire et l'oubli et les processus de vie et de mort (Augé, $2001: 21)^{18}$. Le rituel permet de rendre l'avenir fertile en ce qu'il « consiste à aller chercher et actualiser quelque chose du passé dans un contexte nouveau et différent; mais aussi un aspect sexuel, d'engendrement, que nous pouvons résumer par l'image de la conception d'un souvenir. Autrement dit, on conçoit un souvenir comme on conçoit une idée et comme on conçoit un enfant » (Atlan, $2003: 321$ ).

C'est ce que la circulation et la transmission des noms mettent en forme chez les Kaingang. En effet, les moitiés et les sections possèdent un stock déterminé de noms qui constituent une espèce de patrimoine ancestral. Les noms des défunts sont libérés lors du Kikikoia et peuvent dès lors être attribués à un futur nouveauné. "C'est là que le passé peut devenir futur et le futur passé... » (Atlan, 2003 : 322). C'est ce lien entre passé et futur que l'abandon du Kikikoia problématise dans l'esprit de certains aujourd'hui.

Robert R. CRÉPEAU

Université de Montréal - Département d'anthropologie

Robert.Crepeau@umontreal.ca

\section{Bibliographie}

Albisetti C., Venturelli A.J., (eds.), 1969, Enciclopedia Bororo, vol. 2, Campo Grande, Museu Regional Dom Bosco.

Atlan Henri, 2003, Les étincelles de Hasard. II. Athéisme de l'Écriture, Paris, Seuil.

Augé Marc, 2001, Les formes de l'oubli, Paris, Rivages poche/Petite Bibliothèque.

Baldus H., 1937, Ensaios de Etnologia Brasileira, São Paulo, Companhia Editorial Nacional.

-, 1960, Estórias e Lendas dos Índios. São Paulo, Livraria Literart Editôra.

La Bible de Jérusalem, 1981, Paris, Éditions du Cerf.

La Bible, 2001, Paris-Montréal, Bayard-Médiaspaul (Nouvelle traduction).

Chaumeil Jean-Pierre, 1983, Voir, Savoir, Pouvoir. Le chamanisme chez les Yagua du

Nord-Est péruvien, Paris, Éditions de l'École des hautes études en sciences sociales.

18. «Cette proximité des deux couples - vie et mort, mémoire et oubli - est partout ressentie, exprimée et même symbolisée. » 
CRÉPEAU Robert R., 1988, "Le Chamane achuar: Thérapeutique et socio-politique ", Recherches amérindiennes au Québec, 18/2-3, pp. 101-114.

-, 1994, " Mythe et rituel chez les Indiens Kaingang du Brésil méridional ", Religiologiques, 10, pp. 143-157.

-, 1997, "Les Kaingang dans le contexte des études gé et bororo ", Anthropologie et sociétés, 21/2-3, pp. 45-66.

-, 2006, "'Os Kamé vão sempre primeiro'. Dualismo social e reciprocidade entre os Kaingang ", Anuário Antropológico 2005 (Rio de Janeiro), pp. 9-33.

D’Angelis da Rocha W., Fernandes FokÂE V., 1994, Toldo Imbú: O cacique Conda, os indios do Xapecó e as terras do Imbú, Chapecó, Universidade do Oeste de Santa Catarina, coll. "Série Documento », 3.

Haverroth M., 1997, Um Estudo Etnobotânico: o uso e a classificação das plantas na Área Indígena Xapecó (oeste de SC), Mémoire de maîtrise, Florianópolis, PPGASUniversidade Federal de Santa Catarina.

Hertz R., 1970, "Contribution à une étude sur la représentation collective de la mort ", in R. HeRTz, Sociologie religieuse et folklore, Paris, Presses Universitaires de France, pp. 1-83, [1907].

LÉvi-Strauss Claude, 1964, Le cru et le cuit, Paris, Plon.

-, 1973, "La geste d'Asdiwal ", Anthropologie structurale deux, Paris, Plon, pp. 175-233.

-, 1983, "Mythe et oubli ", in Le regard éloigné, Paris, Plon, pp. 253-261.

Maybury-Lewis D., (ed.), 1979, Dialectical Societies. The Gê and Bororo Indians of Central Brazil, Cambridge, Harvard University Press.

Nimuendajú C., 1993, Ethnografia e Indigenismo, Campinas, Editora da Unicamp [1913].

Pellizzaro S., 1980, Tsunki. Mitos y ritos de la pesca, Sucua, Mundo Shuar.

SANTOS S.C., 1981, "Indigenismo e Expansão Capitalista. Faces da agonia Kaingang ", Cadernos de Ciências Sociais, 2/2, pp. 1-73.

VeIGA J., 1994, Organização social e cosmovisão Kaingang: Uma introdução ao parentesco, casamento e nominação em uma sociedade Jê Meridional, Mémoire de maîtrise, Campinas, PPGAS, Universidade Estadual de Campinas.

-, 2000, "A retomada da festa do Kikikoi no PI Xapecó e a relação desse ritual com os mitos Kaingang ", in L.T. Mota, F.S. Noelli, S.K. Tommasino, (eds.), Uri e Wãxi. Estudos interdisciplinares dos Kaingang, Londrina, Editora UEL, pp. 261-292.

Wilbert J., (ed.), 1978, Folk Literature of the Gê Indians, I, Los Angeles, University of California Press - Latin American Center Publications, coll. "UCLA Latin American Studies ", 44.

Wilbert J., Simoneau K., (eds.), 1984, Folk Literature of the gê Indians, II, Los Angeles, University of California Press-Latin American Center Publications, coll. «UCLA Latin American Studies ", 58. 


\section{Résumé}

Jusqu'à récemment la vie rituelle des Kaingang du Brésil, une société amérindienne de la famille linguistique Gé, comportait un temps fort constitué par la réalisation annuelle du Kikikoia - littéralement "manger l'hydromel »-, un important rite de secondes funérailles. La reprise en 1976 de la réalisation de ce rite, délaissé pendant près de vingt-cinq ans suite à de fortes pressions externes, a permis de lui donner une nouvelle pertinence. En effet, la réactualisation du rituel coïncide avec un regain de vigueur des luttes pour la préservation de la réserve kaingang de Xapecó, dont le territoire initial a été amputé de plusieurs milliers d'hectares au XX $X^{e}$ siècle. Les Kaingang furent tout à fait explicites à ce sujet en liant sans cesse la réalisation du rituel à un devoir de mémoire, c'est-à-dire à la préservation des terres ancestrales très fertiles et convoitées qu'ils possèdent actuellement. Si le rite rappelle ou remémore un acte créateur initial, il ne peut le faire qu'en ne le répétant pas, car un acte créateur ne peut l'être qu'une fois. Ainsi, le devoir de mémoire s'exprimant par le rite ne peut pas être seulement répétition du passé, mais création au présent d'un passé et d'un futur.

Mots-clés : Brésil, société amérindienne, funérailles, rituel, devoir de mémoire.

\section{Abstract}

Until recently the ritual life of the Kaingang of Brazil, an Amerindian society of the Gê linguistic family, was characterized by an elaborated second funeral ceremony: the Kikikoia-literally "eating the honey beer". Following strong external pressures, the Kaingang abandoned its performance during almost 25 years. Its renewal in 1976 by the people of Xapecó Indian Reservation in Santa Catarina is directly related to an intensification of their land claims. The Kaingang were always explicit about this by systematically relating the annual performance of Kikikoia with a duty of memory associated to the recuperation and preservation of their ancestral territory. If ritual is all about remembering, recollecting and reenacting an initial event of creation, nonetheless ritual performance cannot simply repeat this opening act because creation happens only once. Consequently, the duty of memory expressed through ritual is not repetitive of the past, but creation, in the present time, of the past and the future.

Key words: South America, Brazil, Social anthropology, mythology, ritual, social memory, Kaingang.

Hasta un tiempo reciente, la vida ritual de los Kaingang de Brasil, una sociedad amerindia de la familia lingüística Gé, suponía un tiempo fuerte constituido por la realización anual del Kikikoia-literalmente, "comer hidromiel »-, un importante rito de segundos funerales. El hecho que en 1976 este rito fuera retomado, luego de haber sido relegado durante más de 25 años debido a fuerte presiones externas, ha permitido darle una nueva pertinencia. En efecto, la reactualización del ritual coincide con un renovado vigor de las luchas por la preservación de la reserva kaingang de Xapecó, cuyo territorio inicial fue considerablemente reducido durante el siglo XX. Los Kaingang fueron totalmente explícitos al respecto ligando sin cesar la realización del ritual con un deber de memoria, es decir, a la preservación de las tierras ancestrales muy fértiles y envidiadas que poseen actualmente. Si el rito rememora un acto creador inicial, solo puede hacerlo sin repetirlo, dado que un acto creador sólo puede serlo una vez. Así, el deber de memoria se expresa por el rito no puede ser solamente repetición del pasado, sino creación en el presente de un pasado y de un futuro. (Trad. de Véronica Béliveau-Giménez)

Palabras clave : Brasil, sociedad amerincia, funerales, ritual, deber de memoria. 
